\title{
Empleo de Nano-Ferritas dn Bioreactor Anaerobio para la Producción Eficiente de Biogás
}

Use of nano-ferrites in anaerobic bioreactor for the efficient production of biogas

\author{
A. Garrido-Hernández ${ }^{D}$, K. Juárez-Rosas ${ }^{\text {DD } 1}$, R. J. Estrada-Martínez ${ }^{D}{ }^{1}$, G. I. Cerón-Montes ${ }^{\text {ID } 1}$, F. J. Martínez-Valdez ${ }^{\text {iD } 1}$ \\ ${ }^{1}$ Universidad Tecnológica de Tecámac, División de Procesos Industriales. Carretera Federal México-Pachuca Km. 37.5 Predio Sierra Hermosa, Tecámac, Estado de \\ México. C.P. 55740. México.
}

\begin{abstract}
Resumen
Se sintetizaron polvos de ferrita de níquel $\left(\mathrm{NiFe}_{2} \mathrm{O}_{4}\right)$ mediante el método hidrotermal. Se utilizaron como precursores cloruro de hierro y de níquel. Se empleó hidróxido de sodio para modificar el $\mathrm{pH}$ y como agente precipitante. Se evaluaron dos condiciones en el método hidrotermal; agua destilada y una solución de $\mathrm{NaOH}$. Los polvos obtenidos se caracterizaron mediante difracción de rayos X, espectroscopia infrarroja por transformada de Fourier y técnica de difracción láser. El análisis IR mostró bandas características de los enlaces $\mathrm{Fe}^{(\mathrm{III})}-\mathrm{O}-\mathrm{Ni}^{(\mathrm{II})}$ y MO-O situadas a 550 y $490 \mathrm{~cm}^{-1}$. Los patrones de difracción de rayos X revelan que los polvos de ferrita de níquel cristalizan en la fase cúbica. La solución de $\mathrm{NaOH}$ utilizada como disolvente en la reacción hidrotermal promueve una fase secundaria. Los tamaños de las partículas de ferrita de níquel fueron estimados en 20-40 $\mu$ m empleando difracción laser. Se realizó un proceso de digestión anaeróbica y una proyección de eficiencia en la obtención de metano.
\end{abstract}

Palabras Clave:

Hidrotermal, ferritas de níquel, digestión anaeróbica

Abstract

Nickel ferrite $\left(\mathrm{NiFe}_{2} \mathrm{O}_{4}\right)$ powders were prepared by the hydrothermal method. Iron and nickel chloride were used as precursors. Sodium hydroxide was used to modify $\mathrm{pH}$ and as a precipitating agent. The obtained powders were characterized by X-ray diffraction, Fourier Transform Infrared Spectroscopy, and laser diffraction technique. The IR analysis showed the characteristic bands of $\mathrm{Fe}^{(\mathrm{III})}-\mathrm{O}-\mathrm{Ni}^{(\mathrm{II})}$ and MO-O bonds located at 550 and $490 \mathrm{~cm}^{-1}$. X-ray diffraction patterns reveal that nickel ferrite powders crystallize in the cubic phase. The $\mathrm{NaOH}$ solution used as a solvent in the hydrothermal reaction promotes a secondary phase. Nickel ferrite particle sizes were estimated at 20-40 $\mu \mathrm{m}$ using laser diffraction. An anaerobic digestion process was carried out and its efficiency in obtaining methane was projected.

Keywords:

Hydrothermal, nickel ferrites, anaerobic digestion

\section{Introducción}

Las ferritas son óxidos metálicos con estructura de espinela de fórmula general $\mathrm{AB}_{2} \mathrm{O}_{4}$, donde $\mathrm{A}$ y $\mathrm{B}$ son cationes metálicos situados en dos sitios cristalográficos diferentes, tetraédrico (sitio A) y octaédrico (sitio B) (Kefeni y col., 2017). El carácter magnético de las nanopartículas utilizadas en diversas industrias depende fundamentalmente del tamaño, la forma, la pureza y la estabilidad magnética (Gupta y col., 2017). Las ferritas de cobalto y níquel, $\mathrm{MFe}_{2} \mathrm{O}_{4}(\mathrm{M}=\mathrm{Co}, \mathrm{Ni})$, están entre los materiales magnéticos más importantes y se han utilizado ampliamente en aplicaciones electrónicas (Yu y col, 2000). La ferrita de níquel es una ferrita blanda con baja coercitividad magnética, pero con alta resistividad eléctrica, lo que la convierte en un excelente material para los transformadores de potencia en aplicaciones electrónicas y de telecomunicaciones (Zhongli y col., 2008). En los últimos años, existe un interés en la preparación de nanopartículas de ferrita de espinela inversa $\left(\mathrm{NiFe}_{2} \mathrm{O}_{4}\right.$ y $\left.\mathrm{CoFe}_{2} \mathrm{O}_{4}\right)$ de alta calidad (Randhawa, y col., 2005). Estas nanopartículas pueden ser sintetizadas por varios procesos. El convencional y más antiguo es por calcinación de una mezcla de óxidos o carbonatos en un horno a alta temperatura (hasta $2000^{\circ} \mathrm{C}$ ) en tiempos de 2 a $24 \mathrm{~h}$. Este proceso produce partículas micrométricas y un gran gasto de energía. En algunos casos la conversión es incompleta y el material sinterizado requiere de una molienda y una segunda calcinación. Por otro lado, se pueden producir polvos de óxidos uniformes y homogéneos mediante métodos de química suave como el sol-gel, secado por aspersión, co-precipitación, precursor del citrato combustión (Martirosyan y Luss, 2011) y otros. Sin embargo, es necesario desarrollar una nueva ruta para la síntesis

\footnotetext{
*Autor para la correspondencia: fmartinezV@uttecamac.edu.mx

Correo electrónico: agarridoh@uttecamac.edu.mx (Aristeo Garrido Hernández), karenkjr@live.com.mx (Karen Juárez Rosas), restradam@uttecamac.edu.mx (Raziel Jesús Estrada Martínez), gceronm@uttecamac.edu.mx (Genaro Iván Cerón Montes) y fmartinezv@uttecamac.edu.mx (Francisco Javier Martínez Valdez) 
de nuevas microestructuras magnéticas e investigar sus propiedades. En este sentido, el método hidrotermal es una de las herramientas más importantes para el procesamiento de materiales avanzados, debido a sus ventajas en el procesamiento de materiales nanoestructurados para una amplia variedad de aplicaciones tecnológicas (electrónica, optoelectrónica, catálisis, cerámica, almacenamiento de datos magnéticos, biomédica, biofotónica, etc.). Además, el método hidrotermal no sólo ayuda en el procesamiento de nanopartículas monodispersas homogéneas, sino que también actúa como uno de los métodos más atractivos para procesar materiales nanohíbridos y nanocompuestos. El método hidrotermal es una ruta de síntesis prometedora, que permite un mejor control de los parámetros de reacción para obtener materiales puros y homogéneos de tamaños nanométricos.

Por otro lado, los materiales en tamaño nanométricos $(1-100$ nm) como aditivo en los procesos de digestión anaerobia podrían mejorar su rendimiento de manera significativa, debido al cambio de sus propiedades físicas químicas y biológicas en función del área superficial. Estas propiedades únicas de los nanomateriales motivan a investigar los efectos de estos materiales en diversas aplicaciones biológicas como el proceso de digestión anaerobia para desarrollar la producción de energía verde (Baniamerian y col. 2019). Los metales como micronutrientes desempeñan un papel muy importante en el rendimiento y la estabilidad de los digestores anaerobios. El Co y el $\mathrm{Ni}$ participan en el proceso bioquímico de producción de metano. El proceso de la digestión anaerobia se compone de forma secuencial cuatro etapas que puedan ser afectadas por la presencia de nanopartículas. En la hidrólisis, el Fe puede producir un potencial de óxido-reducción $(-0.44 \mathrm{~V})$ por lo que es beneficioso para la reacción de hidrólisis (Velimirovic y col., 2016). Ajay y col. indican que la producción de biogás y el contenido de metano presentan una relación lineal con la concentración de las nanopartículas de níquel (hasta 2 $\mathrm{mg} / \mathrm{l}$ ). El contenido medio de metano del sustrato tratado con 2 $\mathrm{mg} / \mathrm{l}$ de nanopartículas de níquel incrementa la eficiencia $101 \%$ con respecto a la muestra control. Los resultados observados son confirmados por lo reportado por Gustavsson y col., (2013), ya que indican que la presencia de cobalto y níquel en el digestor anaeróbico mejora la generación de biogás y contenido de metano.

En esta investigación se propone la síntesis y caracterización de $\mathrm{NiFe}_{2} \mathrm{O}_{4}$. Se realizarán ensayos de digestión anaerobia finalmente de acuerdo con la literatura se realiza una proyección de la eficiencia que se pueden obtener empleado ferritas de níquel.

\section{Metodología}

Los polvos de ferrita de níquel se prepararon por el método hidrotermal de la siguiente manera: se mezclaron soluciones acuosas de $\mathrm{NiCl}_{2} \cdot 6 \mathrm{H}_{2} \mathrm{O}$ y $\mathrm{FeCl}_{3} \cdot 6 \mathrm{H}_{2} \mathrm{O}$ a $0.5 \mathrm{M}$ en relación de volumen 1:2, seguidas de la adición por goteo de una solución de hidróxido de sodio a $2 \mathrm{M}$ hasta alcanzar un $\mathrm{pH}$ de 11 . Después de ajustar el $\mathrm{pH}$, la solución se mantuvo bajo agitación durante 15 minutos. El precipitado se lavó con agua destilada y se centrifugó a $8000 \mathrm{rpm}$ durante $15 \mathrm{~min}$. Luego se realizaron dos rutas diferentes por el método hidrotermal. En una de ellas, el gel resultante se vertió en un recipiente de Teflon ${ }^{\circledR}$ y luego se añadió un volumen apropiado de solución de $\mathrm{NaOH}(2 \mathrm{M})$ o de agua destilada, después el recipiente de Teflon ${ }^{\circledR}$ se vertió en autoclave de acero inoxidable y se calentó a $170{ }^{\circ} \mathrm{C}$ durante 15 horas. Todas las muestras se lavaron tres veces con agua destilada usando centrifugación. Finalmente, los polvos lavados se secaron a $60{ }^{\circ} \mathrm{C}$ durante 24 horas
La digestión anaerobia se realizó en frascos de $250 \mathrm{ml}$ a escala laboratorio. En el proceso, se utilizó como inoculo lodo granular metanogénico, proveniente de la planta de digestión anaerobia de la Universidad Autónoma Metropolitana-Iztapalapa. Las muestras analizadas fueron: como control el lodo granular solo, así como, la fracción orgánica de los residuos sólidos urbana (FORSU) y como muestra a analizar; la fracción orgánica de los residuos sólidos urbanos más el lodo granular (FORSU+INÖCULO) con una relación inóculo sustrato de sólidos volátiles de 0.5 . Los residuos sólidos orgánicos fueron los generados en la cafetería de la Universidad Tecnológica de Tecámac. Durante la digestión anaerobia se midió el pH y se ajustó la temperatura con un termómetro de mercurio convencional, buscando mantener una temperatura cercana a $\operatorname{los} 36^{\circ} \mathrm{C}$. También, se midió la alcalinidad total para determinar la concentración de carbonatos, bicarbonatos e hidróxidos presentes en las muestras (APHA, 2017). El volumen de biogás se determinó por desplazamiento de columna, la cual, contenía solución salina saturada $300 \mathrm{~g} / \mathrm{L}$ a pH de 2 y como indicador se utilizó rojo de metilo que vira a amarillo cuando se disuelve el $\mathrm{CO}_{2}$. Se determinó la composición del biogás por desplazamiento de columna, la cual, contenía solución de $\mathrm{NaOH}$ $3 \mathrm{M}$ con timoftaleina como indicador (Berrelleza y coll., 2014).

Los análisis FT-IR se obtuvieron en un espectrofotómetro NICOLET Magna-IR TM 550 en modo de transmitancia, la medición se registró en el número de onda de 4000 a $450 \mathrm{~cm}^{-1}$ para estudiar los enlaces característicos en las ferritas de níquel; para este estudio se compactó una mezcla homogénea de polvos de ferrita y $0.25 \mathrm{~g}$ de ioyudor de cesio CsI para obtener pellets de 10 $\mathrm{mm}$ de diámetro. En el análisis de difracción de rayos $\mathrm{X}$ se utilizaron $20 \mathrm{mg}$ de polvos secos en un difractómetro (Siemens D5000) para identificar la fase cristalina de los polvos. La distribución del tamaño de las partículas fue analizada por la técnica de difracción láser usando Beckman Coulter LS 13-320 ATP. En esta medición, los polvos de ferrita se dispersaron en agua desionizada usando un láser de $50 \mathrm{mv}$.

\section{Resultados}

Existe diferencia significativa entre los espectros infrarrojo de la ferrita de níquel obtenida utilizando agua destilada y solución de $\mathrm{NaOH}$ (ver Figura 1) debido a la dificultad de eliminar impurezas durante la etapa de lavado cuando se utiliza la solución de $\mathrm{NaOH}$. En lo que respecta a la literatura, Almeida y col., (2014) informaron de que el procesamiento hidrotermal de la solución precursora mixta de $\mathrm{FeCl}_{3} / \mathrm{NiCl}_{2}$ da lugar a la síntesis de productos de reacción intermedios o primarios en función de la temperatura de reacción; en este experimento. La reacción hidrotermal tuvo lugar a $170{ }^{\circ} \mathrm{C}$ durante 15 horas, lo que indica buenas condiciones de síntesis para la formación de ferrita de níquel.

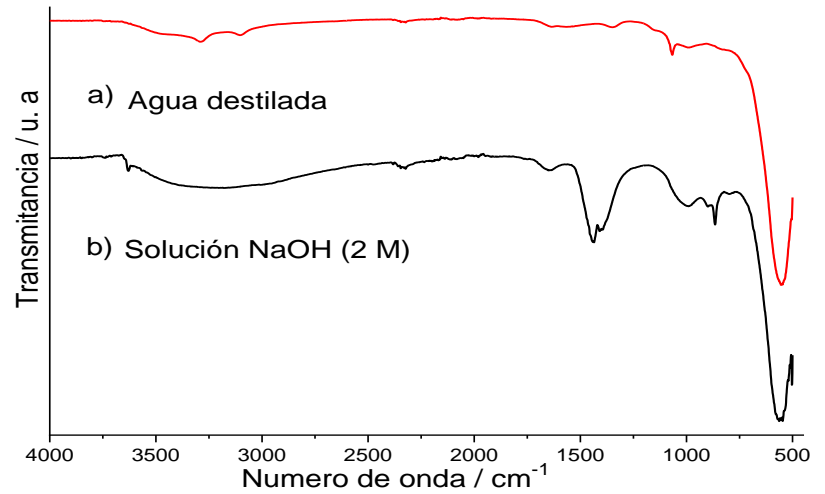

Figura 1 Espectros FT-IR de ferrita de níquel sintetizada por método hidrotermal. 
Las bandas localizadas alrededor de 1430 y $1000 \mathrm{~cm}^{-1}$ se relaciona con el grupo carbonato, el cual se forma mediante la absorción de $\mathrm{CO}_{2}$ de la atmosfera, ya que la solubilidad de este compuesto aumenta significativamente en solución básicas, es bien conocido que la absorción de $\mathrm{CO}_{2}$ en una solución acuosa básica se lleva acabo de la siguiente forma (Bruckman y col., 2013):

$$
\begin{aligned}
& \mathrm{CO}_{2}+\mathrm{H}_{2} \mathrm{O} \leftrightarrow \mathrm{H}_{2} \mathrm{CO}_{3} \\
& \mathrm{H}_{2} \mathrm{CO}_{3} \leftrightarrow \mathrm{HCO}_{3}^{-}+\mathrm{H}^{+} \\
& \mathrm{HCO}_{3}{ }^{-} \leftrightarrow \mathrm{CO}_{3}{ }^{2-}+\mathrm{H}^{+}
\end{aligned}
$$

Es importante mencionar, que cuando la solución es más básica las reacciones 2 y 3 son muy significativas y con esto la posibilidad de formar carbonato de hierro o níquel. Según los datos de la literatura, en el rango de $1000-100 \mathrm{~cm}^{-1}$, las bandas FTIR de los sólidos se asignan normalmente a la vibración de los iones en la red cristalina (Stuart y col. 2004). Estas bandas de absorción se atribuyen normalmente a los enlaces de metaloxígeno. En ferritas con estructura espinela inversa aparecen dos bandas de absorción amplias principales situadas a $555 \mathrm{~cm}^{-1}$ y 490 $\mathrm{cm}^{-1}$, dichas bandas corresponden a las vibraciones de estiramiento intrínsecas del metal en el sitio tetraédrico, (MT-O) y a la vibración de estiramiento del metal octaédrico (MO-O), respectivamente (Nejati y col 2012). En particular, la vibración de estiramiento del metal en el sitio tetraédrico de $\mathrm{Fe}-\mathrm{O}$ en la ferrita de níquel obtenida se sitúan alrededor de $555 \mathrm{~cm}^{-1}$, esta posición difiere con la banda de absorción reportada por Salavati y col., (2009) ya que la vibración de estiramiento de $\mathrm{Fe}-\mathrm{O}$ se localizó en $606 \mathrm{~cm}^{-1}$. Esta diferencia depende de método de síntesis ya que cuando el tamaño de cristalita aumenta las bandas de absorción de $\mathrm{Fe}-\mathrm{O}$ se mueven hacia números de onda más grandes debido al parámetro de la red. Además, es muy conocido que el tratamiento térmico aumenta el tamaño de los cristalita, por lo que se obtiene un mayor tamaño de estos por el método de coprecipitación. Por otro lado, la banda de absorción situada alrededor de $3320 \mathrm{~cm}^{-1}$ indica la presencia de grupos $\mathrm{OH}^{-}$que se atribuye al agua adsorbida.

La Figura 2 muestra los patrones de rayos $\mathrm{X}$ de ferrita de níquel, preparadas por método hidrotermal en agua destilada y una solución de $\mathrm{NaOH}$ al $2 \mathrm{M}$ a $170{ }^{\circ} \mathrm{C}$ durante 15 horas. Las dos muestras tienen picos de cristalización que corresponden a los planos de reflexión (220), (311), (222), (400), (422), (511) y (440) que se asocian a la estructura cúbica (No. JCPDS-01-087-2335) (Salavati-Niasari y col 2009). Los picos de difracción son angostos lo que indican buena cristalinidad de los sistemas.

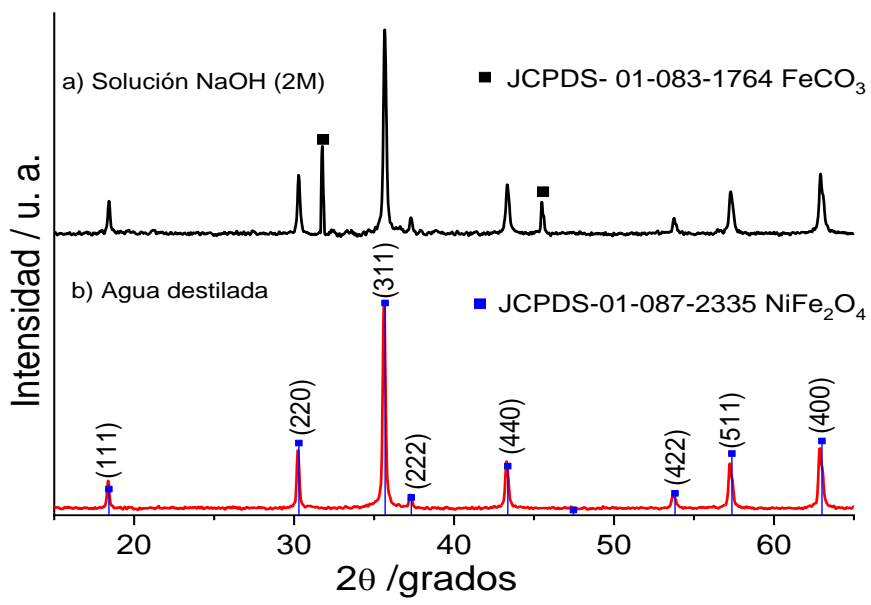

Figura 2 Patrones de rayos $X$ del polvo de ferrita de níquel sintetizado por hidrotermal
Por otro lado, los patrones XRD de la muestra (a) mostrados en la Figura 2 en línea roja revelan una fase secundaria. Este efecto puede estar relacionado con la solubilidad del níquel y los hidróxidos de $\mathrm{Fe}$, ya que según el diagrama de Pourbaix el pH básico promovido por la solución de $\mathrm{NaOH}$ favoreciendo la formación de $\mathrm{Ni}(\mathrm{OH})_{2}$ o $\mathrm{Fe}(\mathrm{OH})_{3}$ por lo que se requiere una alta energía para disolver estas fases. Al existir condiciones ideales de $\mathrm{pH}$ la absorción de $\mathrm{CO}_{2}$, ocasiona que la formación de oxicarbonatos de hierro (fase intermedia), la cual evoluciona a conviertan en carbonato de hierro debido al pH de la solución de $\mathrm{NaOH}$ (13.7). De hecho, los picos localizados a $2 \theta=31.85$ y $45.44^{\circ}$ se asocian a un a los planos cristalinos (104 y 202) del subproducto $\mathrm{FeCO}_{3}$, que corresponde la fase siderita del $\mathrm{FeCO}_{3}$ de acuerdo con la carta de difracción JCPDS 01-083-1764 (Zhen y col., 2016). La fase secundaria evidenció en los análisis de infrarrojo, la cual se formó debido a las condiciones básicas de la reacción.

Se observa que las condiciones de síntesis modifican los parámetros de la red ya que se detecta un desplazamiento de los picos de DRX. Por otro lado, se calculó el tamaño medio de la cristalita se calculó a partir de los picos situados a $2 \theta=60.26^{\circ}$, $35.66^{\circ}, 43.31^{\circ}$ utilizando la fórmula de Scherrer:

$$
D=\frac{k \lambda}{\beta \cos \theta}
$$

Donde, D es el tamaño cristalino promedio, $\mathrm{k}$ la constante de Scherrer (0.9), para las partículas esféricas, $\lambda$ es la longitud de onda de los rayos X, b el ancho de la línea angular de la mitad de la intensidad máxima y $\theta$ es el ángulo de Bragg en grados unidad. Los tamaños de la cristalita se estimaron en 40 y $38 \mathrm{~nm}$ para los polvos de níquel sintetizados en agua destilada y solución de $\mathrm{NaOH}$.

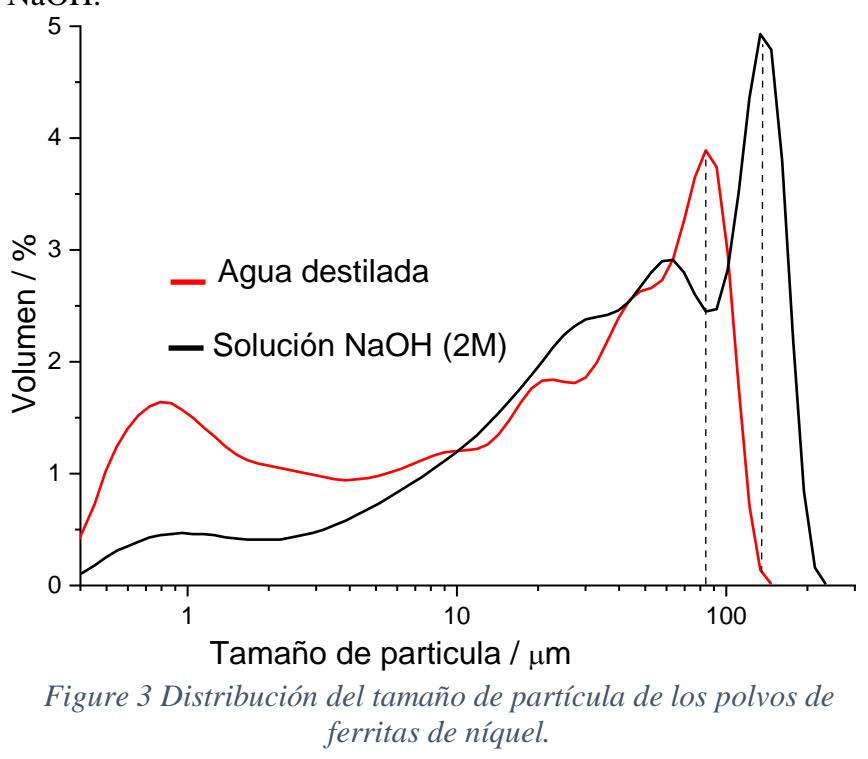

Por otro lado, el tamaño de las partículas de ferrita de níquel se estimó utilizando difracción laser, la estimación del tamaño de las partículas depende del disolvente utilizado durante la reacción hidrotermal debido a que el disolvente cambia la carga superficial de las partículas, es bien sabido que la carga superficial cambia la aglomeración de las partículas. Una mejor dispersión de partículas conlleva a una estimación del tamaño de las partículas más exacta (Kumary col 2011). Para obtener un mejor secado, los polvos de ferrita de níquel obtenidos, mediante el uso de la solución de $\mathrm{NaOH}$ como disolvente llevó más tiempo. Esto indicó un cambio evidente en la carga superficial de las partículas, además de que las ferritas de níquel son magnéticas como resultado se obtiene una distribución de tamaño diferente tal como se muestra en la 
Figura 3. Se observa una distribución de tamaños de partículas multimodal para las ferritas obtenidas mediante las dos rutas hidrotermales; para el agua destilada se localizan tres tamaños característicos $800 \mathrm{~nm}, 20$ y $83 \mu \mathrm{m}$, mientras que para las ferritas sintetizadas usando la solución de $\mathrm{NaOH}$ al $2 \mathrm{M}$ se muestran tamaños de 25, 45 y $105 \mu \mathrm{m}$, respectivamente. La principal desventaja en la determinación del tamaño de las partículas por difracción laser de las partículas magnéticas es su aglomeración, por lo que es necesario un medio adecuado para dispersar las partículas. Por lo tanto, es necesario el uso de surfactante como agente dispersor para aumentar la precisión de la medición del tamaño de las partículas.

En la Figura 4 se muestra la producción de metano durante los diferentes tratamientos dos controles inóculo y FORSU sin inóculo respectivamente donde no se observó una producción de metano considerable y el tratamiento de FORSU+inóculo la cual presento una un rendimiento de metano de $470 \mathrm{ml}$ de metano por sólido volátil ( $\mathrm{NL} \mathrm{CH}_{4} \mathrm{~kg}^{-1} \mathrm{VS}$ de FORSU) a partir del día 31 de digestión. El proceso de digestión anaerobia de FOSU más inóculo y ferritas se muestra en línea roja, la cual es la estimación del efecto que pudiera presentar, es decir, aumentar el rendimiento del proceso anaerobio en un $33 \%$ con respecto al experimento obtenido de acuerdo con la literatura. Abdallah y col., (2019) ha reportado un incremento en el rendimiento de la digestión anaerobia debido a la adición de nanopartículas en especial de nano ferritas. Se han propuesto diferentes mecanismos de acción unos vinculados con la relación área/volumen y otros por efecto del potencial óxido-reducción. Sin embargo, es necesario realizar ensayos con el propósito de investigar las causas (composición y morfología) del incremento en los rendimientos de producción de metano.

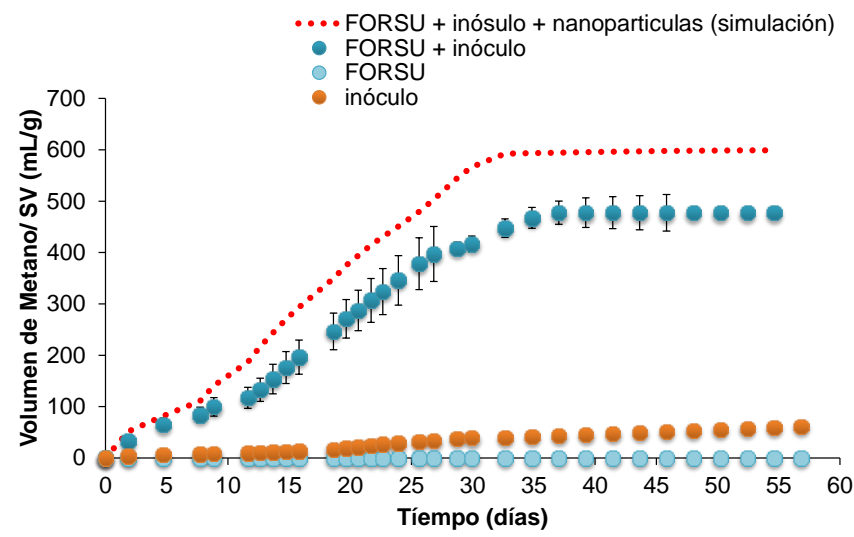

Figura. 4 Volumen de metano durante la digestión anaerobia de residuos orgánicos.

Las concentraciones de ferritas de níquel a utilizar dependerán de la composición y morfologías de las partículas, por ejemplo Abdallah y col., (2019) sintetizaron dos tipos de PN (Ni-Ferrita y $\mathrm{Ni}$-Co-Ferrita) utilizando el método de coprecipitación, añadieron tres concentraciones diferentes $(20,70$ y $130 \mathrm{mg} / \mathrm{l})$ de cada tipo a reactores de biogás separados para estudiar su efecto en la producción de biogás en comparación con el reactor blanco. Los resultados mostraron un aumento de la producción de biogás del $6,6 \%, 5,9 \%$ y $32,9 \%$ al utilizar NPS de Ni-Co-Ferrita en concentraciones de 20, 70 y $130 \mathrm{mg} / \mathrm{l}$ en comparación con el reactor sin nanopartículas, respectivamente. Además, los NPS de Ni-Ferrita lograron mejoras en el biogás de $30.8 \%, 28.5 \%$ y $17.9 \%$ en concentraciones de 20, 70 y $130 \mathrm{mg} / \mathrm{l}$, respectivamente. Por lo tanto, la perspectiva de este trabajo es emplear ferrita de níquel $\left(\mathrm{NiFe}_{2} \mathrm{O}_{4}\right)$ para la producción de metano.

\section{Conclusiones}

Se obtuvieron polvos de ferrita de níquel sintetizados por el método hidrotermal. El uso de una solución de $\mathrm{NaOH}$ al $2 \mathrm{M}$ induce a la formación de fases secundarias debido a la absorción $\mathrm{CO}_{2}$ de la atmosfera durante la agitación de los precursores. La espectroscopia infrarroja confirma los enlaces característicos de la ferrita de níquel cristalizados en la fase cúbica. Los polvos de ferrita de níquel presentaron tamaños de cristalitas muy similares 38 y $40 \mathrm{~nm}$ para los polvos de níquel sintetizados en solución de $\mathrm{NaOH}(2 \mathrm{M})$ y agua destilada, respectivamente. La distribución de tamaño de partícula por difracción laser demostró una distribución multimodal debido a las condiciones de síntesis y propiedades magnéticas de las ferritas de níquel, dando como resultados aglomerados de diversos tamaños. Los tratamientos de digestión anaerobia mostraron la presencia de metano en la producción de biogás producido. Hallazgos en literatura demuestran que la eficiencia en la producción biogás puede mejorarse mediante empleo de ferritas de níquel.

\section{Agradecimientos}

Los autores agradecen el apoyo por uso de instalaciones al centro de cooperación academia industria del Universidad Tecnológica de Tecámac, en especial al Doctor Genaro Iván Cerón-Montes por su compromiso con la ciencia e investigación en la Universidad tecnológica de Tecámac.

\section{Referencias}

Abdallah, M. S., Hassaneen, F. Y., Faisal, Y., Mansour, M. S., Ibrahim, A. M., Abo-Elfadl, S., Salem, H. G., \& Allam, N. K. (2019). Effect of Ni-Ferrite and Ni-Co-Ferrite nanostructures on biogas production from anaerobic digestion. Fuel, 254, 115673 .

Ajay, C. M., Mohan, S., Dinesha, P., \& Rosen, M. A. (2020). Review of impact of nanoparticle additives on anaerobic digestion and methane generation. Fuel, 277, 118234.

Almeida, T. P., Fay, M., Zhu, Y., \& Brown, P. D. (2012). Hydrothermal synthesis and near in situ analysis of $\mathrm{NiFe} 2 \mathrm{O} 4$ nanoparticles. Journal of Nanoscience and Nanotechnology, 12(11), 8797-8800.

Almeida, T. P., Moro, F., Fay, M. W., Zhu, Y., \& Brown, P. D. (2014). Tuneable magnetic properties of hydrothermally synthesised core/shell $\mathrm{CoFe}_{2} \mathrm{O}_{4} / \mathrm{NiFe}_{2} \mathrm{O}_{4}$ and $\mathrm{NiFe}_{2} \mathrm{O}_{4} /$ $\mathrm{CoFe}_{2} \mathrm{O}_{4}$ nanoparticles. Journal of Nanoparticle Research, 16(5), 1.

Baniamerian, Z., Mehdipour, R., \& Murshed, S. S. (2019). An experimental investigation of heat of vaporization of nanofluids. Journal of Thermal Analysis and Calorimetry, 138(1), 645-657.

Bruckman, V. J., \& Wriessnig, K. (2013). Improved soil carbonate determination by FT-IR and X-ray analysis. Environmental chemistry letters, 11(1), 65-70.

de Dios, A. S., \& Díaz-García, M. E. (2010). Multifunctional nanoparticles: Analytical prospects. Analytica chimica acta, 666(1-2), 1-22.

Gupta, N., Jain, P., Rana, R., \& Shrivastava, S. (2017). Current development in synthesis and characterization of nickel ferrite nanoparticle. Materials Today: Proceedings, 4(2), 342-349.

Gustavsson, J., Yekta, S. S., Sundberg, C., Karlsson, A., Ejlertsson, J., Skyllberg, U., \& Svensson, B. H. (2013). 
Bioavailability of cobalt and nickel during anaerobic digestion of sulfur-rich stillage for biogas formation. Applied energy, $112,473-477$.

Kebede K.Kefeni, Bhekie B., MambaTitus A.M.Msagati.(2017).Influence Application of spinel ferrite nanoparticles in water and wastewater treatment.Separation and Purification Technology vol.188 552.

Kumar A., Jacob B., Singh S. y Mohammed E., (2011). «Influence of preparation method on structural and magnetic properties of nickel ferrite nanoparticles,» Bulletin of Materials Science, vol. 34, no 7, pp. 1345-1350.

Lee, D., Rubner, M. F., \& Cohen, R. E. (2006). All-nanoparticle thin-film coatings. Nano letters, 6(10), 2305-2312.

Lucilha, A. C., Silva, M. R. da, Ando, R. A., Dall'Antonia, L. H., \& Takashima, K. (2016). ZNO and AG-ZNO crystals: Synthesis, characterization, and application in heterogeneous photocatalysis. Química Nova, 39(4), 409-414.

Martirosyan, K. S., \& Luss, D. (2011). U.S. Patent No. 7,897,135. Washington, DC: U.S. Patent and Trademark Office.

Mathkar, A., Tozier, D., Cox, P., Ong, P., Galande, C., Balakrishnan, K., Leela Mohana Reddy, A., \& Ajayan, P. M. (2012). Controlled, stepwise reduction and band gap manipulation of graphene oxide. The journal of physical chemistry letters, 3(8), 986-991.

Nejati K. y Zabihi R., (2012). «Preparation and magnetic properties of nano size nickel ferrite particles using hydrothermal method,» Chemistry Central Journal; 2012; 2-6; 6 (23); , vol. 6, no 23, pp. 2-6.

Patil K. C., Aruna S. T. y Ekambaran S., (1997). «Combustion synthesis,» Current Opinion in Solid State and Materials Science, vol. 2, no 1, pp. 158-165.

Randhawa, B. S., Dosanjh, H. S., \& Kaur, M. (2005). Preparation of ferrites from the combustion of metal nitrate-oxalyl dihydrazide solutions.

Ren, D., Ang, B. S.-H., \& Yeo, B. S. (2016). Tuning the selectivity of carbon dioxide electroreduction toward ethanol on oxidederived $\mathrm{Cu} \times \mathrm{Zn}$ catalysts. Acs Catalysis, 6(12), 8239-8247.

Rice, A., Baird, E. W., \& Eaton, R. B. (2017). APHA 2017 Standard Methods for Examination of Water and Wastewater (Washington: American Public Health Association, American Water Works Association, and Water Env. Federation ISBN).
Salavati-Niasari, M., Davar, F., \& Mahmoudi, T. (2009). A simple route to synthesize nanocrystalline nickel ferrite (NiFe2O4) in the presence of octanoic acid as a surfactant. Polyhedron, 28(8), 1455-1458.

Stuart B., (2004). Infrared Spectroscopy: Fundamentals and Applications, England: Wiley. p. 143.

Sugimoto M., (1999). «The Past, Present, and Future of Ferrites,» Journal of the American Ceramic Society, vol. 82, $\mathrm{n}^{\mathrm{o}} 2$, pp. 269-280.

Suresh, K., Kumar, N. R. S., \& Patil, K. C. (1991). A novel combustion synthesis of spinel ferrites, orthoferrites and garnets. Advanced Materials, 3(3), 148-150..

Velimirovic, M., Schmid, D., Wagner, S., Micić, V., von der Kammer, F., \& Hofmann, T. (2016). Agar agar-stabilized milled zerovalent iron particles for in situ groundwater remediation. Science of The Total Environment, 563, 713-723.

Verma, A., Goel, T. C., \& Mendiratta, R. G. (2000). Low temperature processing of NiZn ferrite by citrate precursor method and study of properties. Materials science and technology, 16(6), 712-715..

Videira-Quintela, D., Guillén, F., Montalvo, G., \& Martin, O. (2020). Silver, copper, and copper hydroxy salt decorated fumed silica hybrid composites as antibacterial agents. Colloids and Surfaces B: Biointerfaces, 195, 111216.

Wu, J., Wang, H., Bao, L., Zhong, J., Chen, R., \& Sun, L. (2018). Novel raspberry-like hollow $\mathrm{SiO} 2 @ \mathrm{TiO} 2$ nanocomposites with improved photocatalytic self-cleaning properties: Towards antireflective coatings. Thin Solid Films, 651, 48-55.

Wypych, G. (2017). Handbook of odors in plastic materials. Elsevier.

Yu Yu S. H. y Yoshimura M., (2000). «Direct Fabrication of Ferrite MFe2O4 (M ) Zn, Mg)/Fe Composite Thin Films by Soft Solution Processing,» Chemistry of Materials, vol. 1212, p. 3805-3810.

Yu, S., Wilson, A. J., Kumari, G., Zhang, X., \& Jain, P. K. (2017). Opportunities and challenges of solar-energy-driven carbon dioxide to fuel conversion with plasmonic catalysts. ACS Energy Letters, 2(9), 2058-2070.

Zhongli Zhongli Wang, Xiaojuan Liu, Minfeng Lv, Ping Chai, Yao Liu, and Jian Meng., (2008). Preparation of Ferrite MFe2O4 (M = Co, Ni) Ribbons with Nanoporous Structure and Their Magnetic Properties,» The Journal of Physical Chemistry B, vol. 112, no 36, p. 11292-11297. 